\title{
On the Impact of Strategy and Utility Structures on Congestion-Averse Games
}

\author{
Thomas Voice ${ }^{1}$, Maria Polukarov ${ }^{1}$, Andrew Byde ${ }^{2}$, and Nicholas R. Jennings ${ }^{1}$ \\ 1 School of Electronics and Computer Science, \\ University of Southampton, UK \\ tdv,mp3,nrj@ecs.soton.ac.uk \\ 2 Hewlett-Packard Laboratories, Bristol, UK \\ andrew. byde@hp.com
}

\begin{abstract}
Recent results regarding games with congestion-averse utilities (or, congestion-averse games - CAGs) have shown they possess some very desirable properties. Specifically, they have pure strategy Nash equilibria, which may be found by a polynomial time algorithm. However, these results were accompanied by a very limiting assumption that each player is capable of using any subset of its available set of resources. This is often unrealistic - for example, resources may have complementarities between them such that a minimal number of resources is required for any to be useful. To remove this restriction, in this paper we prove the existence and tractability of a pure strategy equilibrium for a much more general setting where each player is given a matroid over the set of resources, along with the (upper and lower) bounds on the size of a subset of resources to be selected, and its strategy space consists of all elements of this matroid that fit in the given size range. (This, in particular, includes the possibility of having a full matroid, or having a set of bases of a matroid.) Moreover, we show that if a player strategy space in a given CAG does not satisfy these matroid properties, then a pure strategy equilibrium need not exist, and in fact the determination of whether or not a game has a pure strategy Nash equilibrium is NP-complete. We further prove analogous results for each of the congestion-averse conditions on utility functions, thus showing that current assumptions on strategy and utility structures in this model cannot be relaxed anymore.
\end{abstract}

\section{Introduction}

Congestion games - in which self-interested players strategically choose from a common set of resources and derive individual utilities that depend on the total congestion on each resourceare fundamental to a wide range of applications. Examples include resource and task allocation, firm competition for production processes, routing problems, network design, and other kinds of resource sharing scenarios in distributed systems $[8,15,16]$.

Such games are important because Rosenthal [15] showed that they always possess Nash equilibria in pure strategies. This follows by a potential function argument [10], implying that such an equilibrium can be reached in a natural way when players iteratively (and unilaterally) improve their strategies in response to the others' choices. However, such a sequence of (even maximal, or, best) responses may take an exponential number of iterations, as is shown in [4]. In fact, it is PLS-complete to compute a pure strategy equilibrium for general congestion games. Motivated by this fact, much recent effort in algorithmic game theory has gone into study of interesting subclasses of congestion games that are computationally tractable. These, for example, include singleton (or, resource selection) congestion games [5], in which each player is restricted to choosing a single resource, and a more general class of matroid congestion 
games [1], in which players choose among the bases of a matroid over the set of resources. Note, however, that in both cases only strategies (i.e., sets) of the same cardinality are allowed (respectively, 1 or the rank of the matroid).

Congestion games have been extensively studied in a variety of contexts in computer science and economics, giving rise to several extensions of the original model. In particular, the models for local-effect games [6], ID-congestion games [9], player-specific [7] and weighted congestion games [7] — in which a player's payoff is effected by the number, identities or weights of players choosing its selected or neighboring resources - have been considered. However, such games have been constrained to use utility functions that are linear sums with respect to resources, and assumed full reliability and synchronicity of services. More recently, additional generalizations [11-14] dealt with the possibility that resources may fail to execute their assigned tasks, or with the actual order in which the tasks are executed, thus incorporating non-linear and non-additive utility functions in the context of congestion games. These models, however, assumed mutual independence among the resources and imposed particular structures on the players' strategy spaces.

Generalising beyond problem classes with desirable properties raises the important question of developing meaningful criteria that have to be satisfied in order to guarantee that these properties are still present in the generalised model. Given the fact that congestion games have pure-strategy equilibria, we are interested in the question of how far such a sufficient criterion for the existence of pure strategy Nash equilibria can go, and which properties would ensure polynomial complexity of such equilibria.

To this end, Byde et al [2] provided a very general framework that, in particular, includes the abovementioned models of congestion games with faulty or asynchronous resources and player-specific congestion games in the superclass called games with congestion-averse utilities (or, congestion-averse games - CAGs). In a CAG, the payoff of a player is determined by the vector of resource congestion (thus capturing the possibility of mutual dependencies among the resources), via any real-valued function that satisfies certain "congestion-averse" conditions - i.e., monotonicity, submodularity and independence of irrelevant alternatives. The authors proved the existence of a pure strategy Nash equilibrium in these games and provided a polynomial time algorithm for its computation. This result was based on the single profitable move property (SPMP) of these games, implying that a strategy profile is a Nash equilibrium if and only if it is stable under adds, drops or switches with a single resource. The congestionaverse assumptions have been shown to be minimal to guarantee the existence of this property. However, the question of necessity of these assumptions for the existence of a pure strategy Nash equilibrium remained open. Also, though the model of CAGs captures a wide range of important scenarios, it assumes that a player is capable of using any subset of its accessible set of resources, which is unrealistic in many real-life situations, in which, for example, only certain sets of resources are useful in combination.

Our contribution. Given this motivation, we show that the analysis of Byde et al [2] can be generalised even further, towards what we call Matroid Congestion-Averse Games, or MCAGs, in which the set of strategies of each player consists of all the sets within a certain size range from a matroid. This, in particular, includes (but is not restricted to) the possibilities of having a full power set over any subset of resources (as in CAGs [2]), or any other matroid, or having 
a set of elements of a fixed size - for instance, singletons (as in resource selection games [5]) or bases of a matroid (as in matroid congestion games [1]).

For this setting, we prove that all such games have the SPMP and possess at least one equilibrium in pure strategies. We give an algorithm which converges on an equilibrium, with time limits polynomial in the number of players and resources in the game. Essentially, we extend all the previous results on CAGs, weakening the bounds on algorithm running time. We further complete these results by showing that under various relaxations of the matroid or congestionaverse assumptions, these properties are no longer present, and in fact the determination of whether or not a game has a pure strategy Nash equilibrium is NP-complete.

Most of the proofs are omitted, due to space limitations.

\section{Notation and Background}

Consider a congestion setting (or, domain) with a finite set $\mathbf{N}=\{1, \ldots, N\}$ of players and a finite set $\mathbf{R}=\left\{r_{1}, \ldots, r_{R}\right\}$ of resources. A player $i$ 's strategy is to choose a subset of resources from $\mathbf{R}$, and every $N$-tuple of strategies $\sigma=\left(\sigma_{i}\right)_{i \in \mathbf{N}}$ corresponds to an $R$-dimensional congestion vector $h(\sigma)=\left(h_{r}(\sigma)\right)_{r \in \mathbf{R}}$ where $h_{r}(\sigma)$ is the number of players who select resource $r$ (we drop the profile to give $h_{r}$ when it's clear which profile is under consideration). For any player $i \in \mathbf{N}$, its personalised vector of congestion, $h^{i}(\sigma)$, is defined to be a vector in $\mathbb{N}^{R}$ that coincides with $h(\sigma)$ for all the resources that have been selected by $i$ and that has zero entries for all of its unselected resources: that is, $h_{r}^{i}(\sigma)=h_{r}(\sigma)$ if $r \in \sigma_{i}$ and $h_{r}^{i}(\sigma)=0$ otherwise. For a vector $h \in \mathbb{N}^{R}$ the "support" of $h, S(h) \subseteq\{1, \ldots, R\}$, is defined as $\left\{j: h_{r_{j}}>0\right\}$. The utility of player $i$ in a congestion setting is given by a function $U_{i}: \mathbb{N}^{R} \rightarrow \mathbb{R}$ that assigns a real value to a (personalised) vector of congestion. ${ }^{3}$

Games with congestion-averse utilities A utility function is congestion-averse if it (i) monotonically decreases with respect to increasing congestion, (ii) is submodular in that the "better" collection of resources a player uses - the less incentive it has to add new resources, and (iii) is independent of irrelevant alternatives (i.e., if a player "prefers" one resource over another at their current congestion levels, then it still does so no matter what other changes are made to any other resources). Formally, given a strategy profile $\sigma$ and a set of elementary changes (or, single moves) defined on $\sigma$ as follows:

- add $A_{i}(r)$ - player $i$ adds an unselected resource $r: \sigma_{i}^{\prime}=\sigma_{i} \cup\{r\}$,

- drop $D_{i}(r)$ - player $i$ drops a selected resource $r: \sigma_{i}^{\prime}=\sigma_{i} \backslash\{r\}$,

- switch $S_{i}\left(r_{+} \leftarrow r_{-}\right)$-player $i$ switches resources by adding resource $r_{+}$and dropping resource $r_{-}$(note that $\left.S_{i}\left(r_{+} \leftarrow r_{-}\right)=A_{i}\left(r_{+}\right)+D_{i}\left(r_{-}\right)^{4}\right)$,

a utility function $U: \mathbb{N}^{R} \rightarrow \mathbb{R}$ is said to be congestion-averse if it satisfies:

\footnotetext{
${ }^{3}$ Note that the player's utility only depends on the numbers of players choosing each resource but not on their identities - that is, we consider anonymous settings (see [3] for results on approximating equilibria in anonymous games).

${ }^{4}$ Here and in what follows, "+" should be understood to mean sequential execution, read left-to-right. We also use this notation to indicate elementary changes applied to strategy profiles: e.g., $\sigma+D$ denotes a drop applied to profile $\sigma$.
} 
- monotonicity: If $S(h)=S\left(h^{\prime}\right)$ and $\forall r, h_{r} \geq h_{r}^{\prime}$, then $U(h) \leq U\left(h^{\prime}\right)$;

- submodularity: Improving a resource selection by either (i) profitable switches, (ii) extending the set of utilised resources or (iii) reducing congestion on them does not make new adds more profitable, or drops less profitable; likewise, unprofitable switches, deleting the resources or increasing the congestion does not make drops more profitable, or adds less profitable. Equivalently, for any $h, h^{\prime}$ and $h^{\prime \prime}$ such that $|S(h)|=1$ and $S(h) \nsubseteq S\left(h^{\prime}\right), S\left(h^{\prime \prime}\right)$,

$$
U\left(h+h^{\prime}\right)-U\left(h^{\prime}\right) \leq U\left(h+h^{\prime \prime}\right)-U\left(h^{\prime \prime}\right),
$$

if either (i) $\left|S\left(h^{\prime}\right) \backslash S\left(h^{\prime \prime}\right)\right|=\left|S\left(h^{\prime \prime}\right) \backslash S\left(h^{\prime}\right)\right|=1$ and $U\left(h^{\prime}\right) \geq U\left(h^{\prime \prime}\right)$, (ii) $S\left(h^{\prime \prime}\right) \subseteq S\left(h^{\prime}\right)$ and $h_{j}{ }^{\prime \prime}=h_{j}{ }^{\prime}$ for any $j \in S\left(h^{\prime \prime}\right)$, or (iii) $S\left(h^{\prime}\right)=S\left(h^{\prime \prime}\right)$ and $h^{\prime} \leq h^{\prime \prime}$; and

- independence of irrelevant alternatives: If $S_{i}\left(r_{+} \leftarrow r_{-}\right)$is a profitable switch for player $i$ given profile $\sigma$, then it is profitable for $i$ from any other profile $\sigma^{\prime}$ satisfying $r_{-} \in \sigma_{i}^{\prime}, r_{+} \notin \sigma_{i}^{\prime}, h_{r_{-}}(\sigma)=h_{r_{-}}\left(\sigma^{\prime}\right)$ and $h_{r_{+}}(\sigma)=h_{r_{+}}\left(\sigma^{\prime}\right)$.

A congestion-averse game (CAG) is a game in the congestion domain with congestion-averse utility functions, where each player $i \in \mathbf{N}$ has a subset $\mathbf{R}_{i} \subseteq \mathbf{R}$ of $R_{i} \in \mathbb{N}$ accessible resources, and its strategy space, $\Sigma_{i}$, is given by a power set of $\mathbf{R}_{i}$.

\section{Matroid congestion-averse games}

In this section, we extend the model of games with congestion-averse utilities to encompass more general and complex player strategy spaces, which we loosely build on matroids. Before we give a formal definition of such games, we briefly introduce matroids. ${ }^{5}$

Definition 1. A matroid, $M$, is a collection of subsets of some set of elements $X$, with the property that if some $Y \subseteq X$ is in $M$ then all subsets of $Y$ are in $M$. Further, if $V \in M$ is such that $|V|<|Y|$ then there exists some $a \in Y \backslash V$ such that $V \cup\{a\} \in M$.

A matroid congestion-averse game (MCAG) is now defined as a game in the congestion domain with congestion-averse utility functions, over strategy spaces where each player $i$ is given a matroid $M_{i}$ and integers $n_{i} \leq m_{i}$, and its strategy space consists of all the subsets $X \in M_{i}$ such that $n_{i} \leq|X| \leq m_{i}$. More precisely,

Definition 2. An $\boldsymbol{M C A G} \Gamma=\left(\mathbf{N}, \mathbf{R},\left(U_{i}(\cdot)\right)_{i \in \mathbf{N}}\right)$ consists of a set $\mathbf{N}$ of $N \in \mathbb{N}$ players, a set $\mathbf{R}$ of $R \in \mathbb{N}$ resources, and for each player $i$ a matroid $M_{i}$ over $\mathbf{R}$, integers $n_{i} \leq m_{i}$, and a congestion-averse utility function $U_{i}: \mathbb{N}^{R} \rightarrow \mathbb{R}$. The strategy space for each player $i \in \mathbf{N}$ is the set of all the subsets $X \in M_{i}$ satisfying $n_{i} \leq|X| \leq m_{i}$, and the payoff to the player from a combination of strategies $\sigma$ is $u_{i}(\sigma)=U_{i}\left(h^{i}(\sigma)\right)$, where $h^{i}(\sigma)$ is i's personalised vector of congestion as determined by $\sigma$.

Remark 1. Note that our strategy structures cover (but are not restricted to) the possibilities of having a power set over any subset of resources, either including or excluding the empty set (as in CAGs), or any other matroid (full or incomplete), or having a set of elements of a fixed size - for example, a set of singletons (as in resource selection games) or a set of bases of a matroid (as in matroid congestion games).

\footnotetext{
${ }^{5}$ For a detailed discussion of matroids, we refer the reader to [17].
} 
Interestingly, as we show below, the CAG technique based on particular "ladders" of elementary changes, appears to be universal enough to capture the matroid case. Recall, however, that this method builds heavily on several properties, including (i) the single profitable move property, (ii) the existence of a strategy profile which is stable to both switches and adds (and a method to find such), and (iii) the possibility to rank resources by their attraction to a player, that easily follows from the fact that any elementary change is available to any player at any strategy profile. For MCAGs, however, the existence of these properties is not at all obvious. We start with their proofs in the following subsection.

\subsection{Preliminary results}

The single profitable move property. We first show that the matroid congestion-averse games have the SPMP - the single profitable move property, implying that a profile is in equilibrium if and only if it does not admit profitable elementary changes. We begin with a lemma.

Lemma 1. Given a strategy profile of an $M C A G$, a player's strategy is a (strict) best response within the subspace of strategies of the same size, if and only if no (strictly) profitable switch from this strategy is available to the player. Indeed, given a profile $\sigma$, suppose some player $i$ has any alternative strategy $\sigma_{i}^{\prime}$ such that $\left|\sigma_{i}\right|=\left|\sigma_{i}^{\prime}\right|$ and player $i$ would (strictly) prefer $\sigma_{i}^{\prime}$ over $\sigma_{i}$. Then, there is some $r_{+} \in \sigma_{i}^{\prime} \backslash \sigma_{i}$ and $r_{-} \in \sigma_{i} \backslash \sigma_{i}^{\prime}$ such that the switch $S\left(r_{+} \leftarrow r_{-}\right)$is (strictly) profitable for player $i$ at $\sigma$.

We can now prove the single profitable move property for MCAGs.

Theorem 1. Given an MCAG, a strategy profile $\sigma$ is a Nash equilibrium if and only if there are no (maximal) strictly profitable switches, drops or adds.

A strategy profile $\sigma$ is termed as A-stable ( $D$-stable, $S$-stable) if it admits no maximally profitable adds (drops, switches); likewise for AS-stable, DS-stable and so on. Thus, the SPMP states that a profile is in equilibrium if and only if it is ADS-stable.

The SPMP has been used to develop techniques for finding pure strategy equilibria in CAGs and several of their subclasses. These methods used particular dynamics of elementary changes that initialised with a strategy profile which is either AS- or DS-stable. Indeed, since in CAGs a player is allowed to use any subset from its set of accessible resource, the existence of such a profile follows trivially - all the players just play the full or the empty set. However, this strategy may not be available for a general MCAG. Nevertheless, as we shall show, every such a game possesses a strategy profile (or, a "state") which is stable under adds and switches.

Finding an AS-stable state. First, we prove the following theorem.

Theorem 2. Given an $M C A G$, consider $P$ the set of pairs consisting of a single resource and a congestion level on that resource. For each player $i$, there exists a ranking function $V_{i}(\cdot)$ on $P$ such that for any congestion vector $h$ and strategy $\sigma$, if there is a switch $S\left(r_{j} \leftarrow r_{k}\right)$ available to player $i$ then $V_{i}\left(r_{j}, h_{j}+1\right) \leq V_{i}\left(r_{k}, h_{k}\right)$ if and only if the switch $S\left(r_{j} \leftarrow r_{k}\right)$ is profitable.

Corollary 1. Any best response dynamics within the reduced space where each player is restricted to the maximal size strategies, will terminate in an $A S$-stable state within $N^{2} R^{2}$ moves. 
Proof. Given an MCAG, we create a congestion game by restricting each player $i$ to the strategies in $M_{i}$ with $m_{i}$ elements, and setting player $i$ 's utility as the sum of $V_{i}(\cdot)$ for resources selected by player $i$. Now, clearly this congestion game has the SPMP, as does the original MCAG, and furthermore, any profitable switch in the derived congestion game is a profitable switch in the original MCAG. Moreover, Lemma 1 implies that any best response move in the derived congestion game corresponds to a best response in the original MCAG, within the reduced strategy space. This means that a pure strategy Nash equilibrium in the derived congestion game will also be an AS-stable pure strategy in the original MCAG. The corollary then follows from the results in [1].

Remark 2. In games with fixed size strategies - like singleton or matroid congestion gamesdrops are never available. So, any AS-stable strategy profile is also a Nash equilibrium. For such games, the above corollary proves the existence of, and provides a method for finding, a pure strategy Nash equilibrium.

Dynamics. Given the existence of an AS-stable state, we now explore the convergence for matroids of the drop- and swap-dynamics, as defined in [2]. We start with a brief definition of drop- and swap-ladders, and then proceed and describe their properties in MCAGs.

Definition 3. A drop ladder is a sequence $D_{i_{0}}\left(r_{0}\right)+S_{i_{1}}\left(r_{1}^{\prime} \leftarrow r_{1}\right)+\cdots+S_{i_{m}}\left(r_{m}^{\prime} \leftarrow r_{m}\right)$, consisting of a maximally profitable drop followed by a sequence of $m \geq 0$ maximally profitable switches, and a swap ladder is a drop-ladder followed by a maximally profitable add with its tail: $D_{i_{0}}\left(r_{0}\right)+S_{i_{1}}\left(r_{0} \leftarrow r_{1}\right)+\cdots+S_{i_{m}}\left(r_{m-1} \leftarrow r_{m}\right)+A_{i_{m+1}}\left(r_{m}\right)$. The swap ladder is described as minimal if all intermediate strategy profiles before the last add were A-stable (i.e., if the add is performed at the first opportunity).

Note that the above definition of a swap ladder implies that a profitable add is made to the tail of the corresponding drop ladder. This is well defined by the following lemma.

Lemma 2. Given a CAG, let $\sigma$ be an AS-stable strategy profile that possesses a drop ladder of length $m$, and let $\sigma^{k}$ denote the result of applying the drop and the first $k$ switches to $\sigma$. Suppose further that for each $\sigma^{k}$ for $1 \leq k<m$ there are no profitable adds. Then, for all $1 \leq k \leq m$, the only switches which are profitable at $\sigma^{k}$ are those which "chain" with the previous switch or the initial drop, i.e. those who switch in the resource which was most recently dropped or switched out. Furthermore, if there is a profitable add $A_{i}\left(r_{+}\right)$for profile $\sigma^{m}$ then $r_{+}=r_{m}$.

Thus, the result of a swap ladder possesses the same congestion vector as the original profile; as we shall see, this will imply that minimal swap ladders preserve AS-stability. The result will follow from Lemma 3 below:

Lemma 3. Consider the sequence of adds, drops and switches that a single player makes in a sequence of minimal swap ladders. For each player, we rank the resources according to the ranking function defined in Theorem 2, using the fixed congestion levels present between swap ladders. Then, (i) if the ranks of resources a player has selected are put in decreasing order, then this set of values increases lexicographically with every switch; (ii) every add must add a resource that is strictly higher ranked than the resource most recently dropped; (iii) the ranks of dropped resources are non-decreasing. 
Corollary 2. There can be no more than $N R(R+2)$ elementary changes in total in any sequence of minimal swap ladders.

Proof. For a given player $i$ with strategy $\sigma_{i}$, let us denote the multiset of ranks of resources in $\sigma_{i}$, according to the personal ranking of player $i$, as $K_{i}$. We extend $K_{i}$ to be an $R$ element multiset by adding $R-\left|\sigma_{i}\right|$ entries of a rank 0, which we formally define as being below all other ranks. Let $V\left(K_{i}\right)$ be the values of $K_{i}$ listed in decreasing order.

Through a series of swap ladder moves, we allow $K_{i}$ to change as the strategy of player $i$ changes. If player $i$ performs a switch, then the rank in $K_{i}$ corresponding to the switched out resource is replaced with the rank of the switched in resource. If player $i$ performs a drop, then all elements of $K_{i}$ which do not refer to resources in the current strategy, are set equal to the rank of the dropped resource. If player $i$ adds a resource, then one of the elements of $K_{i}$ which no longer refers to a resource in player $i$ 's strategy is changed to refer to the rank of the new resource. Lemma 3 ensures that each of these moves causes an increase in the lexicographical rank of the values of $V\left(K_{i}\right)$.

Now, $K_{i}$ has $R$ elements taken from a set of $R+1$ ranks, so there can be at most $R(R+1) / 2$ strict increases in lexicographical rank of $V\left(K_{i}\right)$. Every swap ladder action causes a strict increase, apart from drops. There cannot be $R$ more drops than there are adds. Thus, the total number of swap ladder actions possible is bounded above by $R$ plus twice $R(R+1) / 2$.

\subsection{Main Results}

We are now ready to conclude the existence and tractability of a pure strategy Nash equilibrium in MCAGs. The following proposition, coupled with Corollary 2, implies our main result.

Proposition 1. Applying a minimal swap ladder to an AS-stable state preserves AS-stability.

Theorem 3. Every MCAG has a pure strategy Nash equilibrium.

Proof. We have shown there is at least one AS-stable strategy profile. If we continually apply minimal swap ladders to an AS-stable strategy profile, then, since the number of minimal swap ladder moves is bounded, we will eventually reach an AS-stable strategy profile to which no minimal swap ladder may be applied. If this AS-stable strategy profile is not D-stable, then we can apply a maximal drop ladder. Since the drop ladder is maximal, the resulting state is S-stable. Furthermore, the resulting state, and every intermediate state along the drop ladder must be A-stable because there were no swap ladders which could be applied to the starting AS-stable state. As long as we have an AS-stable profile which is not D-stable, we can repeat this process. Every time we do so, we reduce the total congestion by one, and thus we can only repeat the process at most $N R$ times. Once we can no longer apply a minimal swap ladder or a maximal drop ladder, we have a pure strategy Nash equilibrium.

\section{Necessity of the MCAG model assumptions}

In this section we complete our results on congestion-averse games, by demonstrating that the strategy space and utility function assumptions in our model cannot be further relaxed. Specifically, we will show that if any of the (ranged) matroid or the congestion-averse properties is removed, a pure strategy equilibrium is not guaranteed to exist, and in fact the determination of whether or not a game has such an equilibrium is NP-complete. 


\subsection{Non-matroid congestion-averse games}

In an MCAG, each player has a strategy space which consists of all the sets within a certain size range from a matroid. This can be expressed equivalently with the following.

Suppose a player has a strategy space $S$. Then, for all $X \neq Y$ in $S$, the following two assumptions hold: (i) if $|X|=|Y|$ then for each element $x \in X$ there is an element $y \in Y$ such that $(X \backslash\{x\}) \cup\{y\} \in S$; (ii) if $|X|<|Y|$ then all $|X|$ element subsets of $Y$ are in $S$. This is termed as the ranged matroid property.

We will show that if we relax any of these assumptions then a pure strategy equilibrium is no longer guaranteed and, in fact, the determination of whether or not a pure strategy equilibrium exists is not tractable. This follows by a reduction argument using the 3-SAT problem which is well known to be NP-complete.

Theorem 4. In an MCAG setting, violation of either of the ranged matroid assumptions on strategy spaces may result in a game with no pure strategy equilibria. Moreover, it is in general NP-complete to determine whether a game possesses such an equilibrium.

\subsection{Non-congestion-averse utilities}

Here we present similar results on the congestion-averseness assumptions on utility functions. Specifically, we show that violation of any of these assumptions (or even partial relaxation of submodularity) may result in a game with no pure strategy equilibrium, and reduce from the 3-SAT to show NP-hardness of the equilibrium existence decision problem.

Theorem 5. In an $M C A G$ setting, if any one of the congestion-averse conditions on utility functions is violated then a pure strategy Nash equilibrium is not guaranteed to exist. Moreover, there are instances of games in which it is NP-complete to determine its existence. This also applies to the case in which the submodularity assumption is only partially violated, that is either parts (i) and (iii) or part (ii) of the assumption hold.

\section{Conclusions}

We investigated the impact of the strategy space and payoff function structures on games with congestion-averse utilities. We extended the previous positive results on the existence and tractability of pure strategy equilibria to the case, in which the set of strategies of each player consists of all the sets within a certain size range from a matroid. This covers a wide range of settings, including those where the players' strategies are represented by singletons, bases of matroids, and power sets over a set of accessible resources. Our result is tight in that the relaxation of the (ranged) matroid property or each of the congestion-averseness conditions may lead to a game without a pure strategy equilibrium, and it is in general NP-complete to determine the existence of such an equilibrium. Thus, we conclude that the current assumptions on strategy and utility structures in this model cannot be further relaxed. 


\section{References}

1. H. Ackermann, H. Röglin, and B. Vöcking. On the impact of combinatorial structure on congestion games. Journal of the ACM, 55(6), 2008.

2. A. Byde, M. Polukarov, and N. R. Jennings. Games with congestion-averse utilities. In SAGT-09, pages 220-232, 2009.

3. C. Daskalakis and C. Papadimitriou. Computing equilibria in anonymous games. In FOCS-0\%, pages 83-93, 2007.

4. A. Fabrikant, C. Papadimitriou, and K. Talwar. The complexity of pure Nash equilibria. In STOC-04, pages 604-612, 2004.

5. S. Ieong, R. McGrew, E. Nudelman, Y. Shoham, and Q. Sun. Fast and compact: A simple class of congestion games. In $A A A I-05$, pages 489-494, 2005.

6. K. Leyton-Brown and M. Tennenholtz. Local-effect games. In IJCAI-03, pages 772-780, 2003.

7. I. Milchtaich. Congestion games with player-specific payoff functions. Games and Economic Behavior, 13:111-124, 1996.

8. I. Milchtaich. Congestion models of competition. American Naturalist, 147(5):760-783, 1996.

9. D. Monderer. Solution-based congestion games. Advances in Math. Economics, 8:397-407, 2006.

10. D. Monderer and L.S. Shapley. Potential games. Games and Economic Behavior, 14:124-143, 1996.

11. M. Penn, M. Polukarov, and M. Tennenholtz. Congestion games with failures. In EC-05, pages 259-268, 2005.

12. M. Penn, M. Polukarov, and M. Tennenholtz. Congestion games with load-dependent failures: Identical resources. Games and Economic Behavior, 67(1):156-173, 2009.

13. M. Penn, M. Polukarov, and M. Tennenholtz. Random order congestion games. Mathematics of Operations Research, 2009 (to appear).

14. M. Penn, M. Polukarov, and M. Tennenholtz. Taxed congestion games with failures. Annals of Mathematics and Artificial Intelligence, 2009 (to appear).

15. R.W. Rosenthal. A class of games possessing pure-strategy Nash equilibria. International Journal of Game Theory, 2:65-67, 1973.

16. T. Roughgarden and E. Tardos. How bad is selfish routing. Journal of the ACM, 49(2):236-259, 2002.

17. A. Schrijver. Combinatorial optimization: Polyhedra and efficiency, volume B, Matroids, Trees, Stable Sets, chapter 36-39. Springer, 2003. 